\title{
EIGENFILTERS FOR THE DESIGN OF SPECIAL TRANSFER FUNCTIONS WITH APPLICATIONS IN MULTIRATE SIGNAL PROCESSING
}

\author{
T. Q. Nguyen ${ }^{\ddagger}$, T. Saramaki* and P. P. Vaidyanathan ${ }^{\ddagger}$ \\ $\ddagger$ Dept. EE, Caltech, Pasadena, CA 91125 \\ D3.8
}

${ }^{*}$ Dept. EE, Tampere Univ. Tech., Finland

\begin{abstract}
Based on the multistage eigenfilters approach, we present a design procedure for finding a spectral factor of an $m$-th band filter and for designing multistage decimation filters. The proposed design method finds spectral factors of $m$-th band FIR filters without direct computation, and yields filters with much higher attenuation than would be possible by conventional methods. Such $m$-th band filters find applications in filter-bank designs, including perfect reconstruction systems.
\end{abstract}

\section{Introduction ${ }^{1}$}

Let $G(z)$ be a nonnegative valued $m$-th band FIR filter. Its spectral factor $\hat{G}(z)$ where $G(z) \triangleq \hat{G}(z) \hat{G}\left(z^{-1}\right)$ finds many applications in multirate signal processing [24], and in QMF banks [5-7]. Designing the nonlinear phase FIR transfer function $G(z)$ with high attenuation often encounters problems if conventional spectral factorization [8] is used. In this paper, an approach is outlined to overcome this difficulty. We shall show that the design of $\hat{G}(z)$ can be done directly using the eigenfilters approach [1], in such a way that its stopband energy is minimized. There is no need to directly find high order spectral factors in the design process. The second application which will be emphasized is the design of multistage decimation filters [3] using eigenfilters. These multistage filters are used to implement decimation filters efficiently.

Bold faced letters denote both vectors and matrices in this paper. Superscript $\left(^{*}\right)$ stands for complex conjugation and $\hat{G}(z)$ denotes the spectral factor of $G(z)$.

\section{Eigenfilters - A review.}

Let $H_{0}(z)$ be the amplitude response of a linear phase lowpass FIR filter of order $\ell-1$ and cutoff frequencies $\omega_{p}$, $\omega_{S}$. We consider the case where $\ell-1$ is even (the odd case can be easily handled). Define the stopband error of $H(z)$ to be $E_{S}=\int_{\omega_{S}}^{\pi}\left|H_{0}\left(e^{j \omega}\right)\right|^{2} d \omega$. Similarly, the passband error is formulated as $E_{p}=\int_{0}^{\omega_{p}}\left|H_{0}\left(e^{j 0}\right)-H_{0}\left(e^{j \omega}\right)\right|^{2} d \omega$ and the total error of $H(z)$ is thus

$$
E=(1-\alpha) E_{p}+\alpha E_{S} .
$$

${ }^{1}$ Work supported in part by the National Science Foundation grants DCI 8552579 and MIP 8604456.
Let us rewrite $H_{0}(z)$ in a more convenient form using the sequence $b(n)$, defined as

$$
b(n)= \begin{cases}2 h((\ell-1) / 2-n), & n \neq 0 \\ h((\ell-1) / 2), & n=0\end{cases}
$$

Here, $h(n)$ is the filter coefficient of $H(z)$. The magnitude response of $H_{0}(z)$ is compactly represented as

$$
H_{0}(z)=\mathrm{b}^{T} \mathrm{c}(\omega)
$$

where $\mathbf{b}=\left[\begin{array}{llll}b(0) & b(1) & \ldots & b(K-1) b(K)\end{array}\right]^{T}, \mathbf{c}(\omega)=[1$ $\cos \omega \cos 2 \omega \ldots \cos K \omega]^{T}$ and $K=\frac{\ell-1}{2}$. Writing in terms of $\mathbf{b}$ and $\mathbf{c}(\omega)$, the total energy in (1) is $E=\mathbf{b}^{T} \mathbf{P b}$ where

$$
\mathbf{P}=(1-\alpha) \int_{0}^{\omega_{p}}(1-\mathbf{c})(1-\mathbf{c})^{T} d \omega+\alpha \int_{\omega_{S}}^{\pi} \mathbf{c c}^{T} d \omega
$$

and $1=\left[\begin{array}{llll}1 & 1 & \ldots & 1\end{array}\right]^{T}$. The minimization requirement in (1) is now equivalent to finding a vector $b$ such that $E$ is minimized where $P$ in (4) is a real, symmetric and positive definite matrix. By Rayleigh principle [9], the eigenvector associated with the smallest eigenvalue of $P$ minimizes the total error $E$. In summary, given $\ell-1, \omega_{p}, \omega_{\mathcal{S}}$ and $\alpha$, the eigen filter coefficients can be obtained from the eigenvector corresponding to the smallest eigenvalue of $\mathbf{P}$. The eigenfilter approach can be extended to incorporate weighting functions on the total error $E$. Consider the following total weighted error $E=\mathbf{b}^{T} \mathbf{P b}$ where

$$
\begin{array}{r}
\mathbf{P}=(1-\alpha) \int_{0}^{\omega_{p}} W_{p}\left(e^{j \omega}\right)(1-\mathbf{c})(1-\mathbf{c})^{T} d \omega \\
+\alpha \int_{\omega_{S}}^{\pi} W_{S}\left(e^{j \omega}\right) \mathbf{c c}^{T} d \omega .
\end{array}
$$

We still can apply Rayleigh principle and obtain the eigenfilter which minimizes the total weighted error as long as $W_{p}\left(e^{j \omega}\right)$ and $W_{S}\left(e^{j \omega}\right)$ are nonnegative valued functions. As elaborated further in [1], eigenfilters design procedure can incorporate both time and frequency domain specifications. Thus, Nyquist, $m$-th band, equiripple, maximally flat, and multiband linear phase FIR filters can all be designed using the weighted eigenfilters approach (abbreviated as the WE approach). 


\section{Spectral Factors of $m$-th band FIR filters.}

Let $G(z)$ be an even order linear phase FIR $m$-th band filter with order $\ell-1$ and real coefficients $g(n)$. To avoid the case where $g(0)=g(\ell-1)=0$, we assume that $\ell-1$ is not an integer multiple of $2 \mathrm{~m}$. Since $G(z)$ is a linear phase filter, its zeros on the unit circle occur in conjugate pairs whereas its zeros on the real axis occur in reciprocal pairs and its zeros neither on the unit circle nor on the real axis occur in reciprocal conjugate quadruples. To be able to have a spectral factor, $G\left(e^{j \omega}\right)$ has to be a nonnegative valued function. Consequently, its zeros on the unit circle have to be double zeros. In other words, if $z_{0}$ is a zero of $G(z)$ on the unit circle, then both $z_{0}$ and $z_{0}^{*}$ are double zeros. On the other hand, if $z_{0}$ is a zero neither on the unit circle nor on the real axis, then $z_{0}^{*}, z_{0}^{-1}$ and $\left(z_{0}^{-1}\right)^{*}$ are also zeros of $G(z)$. Similarly, $z_{0}$ and $z_{0}^{-1}$ is a pair of zeros on the real axis. Let's group the zeros on the unit circle into $G_{1}^{2}(z)$ and the zeros not on the unit circle into $G_{0}(z)$. We then have $G(z)=G_{0}(z) G_{1}^{2}(z)$, so that the spectral factor $\hat{G}(z)$ is $\hat{G}_{0}(z) G_{1}(z)$ where $G_{0}(z)$ is the spectral factor of $G_{0}(z)$. The problem statement is thus as follows: Find $\hat{G}(z)$ such that

1. $G(z)=\hat{G}(z) \hat{G}\left(z^{-1}\right)$ is an $m$-th band filter and

2. $E=\int_{\omega_{S}}^{\pi}\left|\hat{G}\left(e^{j \omega}\right)\right|^{2} d \omega$ is minimized.

Minimizing $E$ in step 2 above is the same as minimizing

$$
E=\int_{\omega_{S}}^{\pi}\left|G\left(e^{j \omega}\right)\right| d \omega=\int_{\omega_{S}}^{\pi}\left|G_{0}\left(e^{j \omega}\right)\right|\left|G_{1}\left(e^{j \omega}\right)\right|^{2} d \omega .
$$

We now discuss an iterative procedure to design the $m$-th band filter $G(z)$ and its spectral factor $\hat{G}(z)$. The iterative procedure works as follows:

1. Initialize $G_{0}\left(e^{j \omega}\right)$ to be unity. Find $G_{1}\left(e^{j \omega}\right)$ such that $\int_{\omega_{S}}^{\pi}\left|G_{1}\left(e^{j \omega}\right)\right|^{2} d \omega$ is minimized using the eigenfilter method.

2. Fix $G_{1}(z)$ and readjust $G_{0}(z)$ by solving a set of linear equations, so that $G(z)=G_{0}(z) G_{1}^{2}(z)$ has $m$ th band property [2].

3. By fixing $G_{0}(z)$ to be the solution obtained in step 2 , find $G_{1}(z)$ using the WE approach such that

$$
E=\int_{\omega_{S}}^{\pi}\left|G_{0}\left(e^{j \omega}\right)\right|\left|G_{1}\left(e^{j \omega}\right)\right|^{2} d \omega \text { is minimized. }
$$

4. If the resulting $G_{0}(z) G_{1}^{2}(z)$ is not satisfacting, go to step 2.

Very few repetitions of these steps results in excellent design. Trivial solutions to the minimization steps can be avoided by constraining the total energy of $G_{1}(z)$ to be unity. Even though the passband error of $G(z)$ does not enter the error function in (6), the passband of $G(z)$ comes out to be good because of the $m$-th band property of $G(z)$. The order of $G_{0}(z)$ is typically much smaller than that of
$G(z)$, and moreover it has no zeros on the unit circle, so it is a simple matter to find a spectral factor $\hat{G}_{0}(z)$ of $G_{0}(z)$ and obtain $\hat{G}(z)=\hat{G}_{0}(z) G_{1}(z)$. The readjustment of $G_{0}(z)$ in step 2 is elaborated here using $m$-th band property.

Let $2 \ell_{0}, \ell_{1}$ and $\ell-1$ be the orders of $G_{0}(z), G_{1}(z)$ and $G(z)$. Thus, $G(z)$ and $\hat{G}(z)$ have orders $2\left(\ell_{0}+\ell_{1}\right)$ and $\left(\ell_{0}+\ell_{1}\right)$ respectively. Denote the filter coefficients of $G_{0}(z), G_{1}(z), G_{1}^{\prime}(z)\left(\triangleq G_{1}^{2}(z)\right)$ and $G(z)$ by $g_{0}(n), g_{1}(n)$, $g_{1}^{\prime}(n)$ and $g(n)$ respectively. As mentioned earlier, $\ell_{0}+$ $\ell_{1} \neq k m$ for any positive integer $k$. Since $G(z)$ is required to be an $m$-th band filter, every $m^{\text {th }}$ coefficient from the mid-point has to be zero, i.e.,

$$
\left\{\begin{array}{l}
g\left(\frac{\ell-1}{2}-k m\right)=g\left(\ell_{0}+\ell_{1}-k m\right)=0, \quad k>0 \\
g\left(\ell_{0}+\ell_{1}\right)=\frac{1}{m} .
\end{array}\right.
$$

The number of known coefficients in $G(z)$ is therefore

$$
L=\left\lfloor\frac{\ell}{m}\right\rfloor=\left\lfloor\frac{2\left(\ell_{0}+\ell_{1}\right)+1}{m}\right\rfloor \text {. }
$$

In terms of filter coefficients, $G(z)=G_{0}(z) G_{1}^{2}(z)=G_{0}(z)$. $G_{1}^{\prime}(z)$ is equivalent to

$$
g(n)=\sum_{k=0}^{2 \ell_{0}} g_{0}(k) g_{1}^{\prime}(n-k) .
$$

For a given filter $G_{1}^{\prime}(z)$, there are $\left(2 \ell_{0}+1\right)$ unknown coefficients of $G_{0}(z)$ and $L$ fixed values of $g(n)$. Consequently, if $L=2 \ell_{0}+1$, then we can uniquely solve for $g_{0}(n)$ in terms of $g_{1}^{\prime}(n)$ such that $(7)$ holds. In other words, $g_{0}(n)$ is uniquely determined in

$$
\begin{gathered}
\left(\begin{array}{ccccc}
\ddots & \vdots & \vdots & \vdots & . \\
\cdots & g_{1}^{\prime}\left(\ell_{1}-m+1\right) & g_{1}^{\prime}\left(\ell_{1}-m\right) & g_{1}^{\prime}\left(\ell_{1}-m-1\right) & \ldots \\
\cdots & g_{1}^{\prime}\left(\ell_{1}+1\right) & g_{1}^{\prime}\left(\ell_{1}\right) & g_{1}^{\prime}\left(\ell_{1}-1\right) & \ldots \\
\cdots & g_{1}^{\prime}\left(\ell_{1}+m+1\right) & g_{1}^{\prime}\left(\ell_{1}+m\right) & g_{1}^{\prime}\left(\ell_{1}+m-1\right) & \ldots \\
. & \vdots & \vdots & \vdots & \ddots
\end{array}\right) \\
\cdot\left(\begin{array}{c}
\vdots \\
g_{0}\left(\ell_{0}-1\right) \\
g_{0}\left(\ell_{0}\right) \\
g_{0}\left(\ell_{0}+1\right) \\
\vdots
\end{array}\right)=\left(\begin{array}{c}
\vdots \\
g\left(\ell_{1}-m+\ell_{0}\right) \\
g\left(\ell_{1}+\ell_{0}\right) \\
g\left(\ell_{1}+m+\ell_{0}\right) \\
\vdots
\end{array}\right)=\left(\begin{array}{c}
\vdots \\
0 \\
\frac{1}{m} \\
0 \\
\vdots
\end{array}\right)(10)
\end{gathered}
$$

Using (8) in $L=2 l_{0}+1$, we have

$$
\left\lfloor\frac{2\left(\ell_{0}+\ell_{1}\right)+1}{m}\right\rfloor=2 \ell_{0}+1 .
$$

Equivalently,

$$
2 \ell_{0}+1 \leq \frac{2\left(\ell_{0}+\ell_{1}\right)+1}{m}<2 \ell_{0}+2
$$

$2 \ell_{0}(m-1)+(m-1) \leq 2 \ell_{1}<2 \ell_{0}(m-1)+(2 m-1)$. 
As long as the order of $G_{1}^{\prime}(z)$ (which is $2 \ell_{1}$ ) satisfies (13), we can always solve equation $(10)$ for $g_{0}(n)$. (10) can be further simplified by observing that $g(n), g_{0}(n)$ and $g_{1}^{\prime}(n)$ are symmetric sequences. Thus, only the first $\frac{L+1}{2}$ equations are sufficient to describe (10), i.e.,

$$
\begin{gathered}
\left(\begin{array}{ccc}
g_{1}^{\prime}\left(\ell_{1}-m \ell^{\prime}+\ell_{0}\right) & \cdots & g_{1}^{\prime}\left(\ell_{1}-m \ell^{\prime}\right) \\
+g_{1}^{\prime}\left(\ell_{1}-m \ell^{\prime}-\ell_{0}\right) & & \\
\vdots & \ddots & \vdots \\
2 g_{1}^{\prime}\left(\ell_{1}+\ell_{0}\right) & \cdots & g_{1}^{\prime}\left(\ell_{1}\right)
\end{array}\right) \cdot\left(\begin{array}{c}
g_{0}(0) \\
\vdots \\
g_{0}\left(\ell_{0}\right)
\end{array}\right) \\
=\left(\begin{array}{c}
g\left(\ell_{1}-m \ell^{\prime}+\ell_{0}\right) \\
\vdots \\
g\left(\ell_{1}+\ell_{0}\right)
\end{array}\right)=\left(\begin{array}{c}
0 \\
\vdots \\
\frac{1}{m}
\end{array}\right)
\end{gathered}
$$

where $\ell^{\prime}=\frac{\ell-1}{2}$. (14) can be compactly rewritten as

$$
\mathbf{A g}_{0}=\mathbf{d}
$$

where $\mathbf{g}_{0}=\left[\begin{array}{llll}g_{0}(0) & \ldots & g_{0}\left(\ell_{0}\right)\end{array}\right]^{T}, \mathbf{d}=\left[\begin{array}{llll}0 & \ldots & \frac{1}{m}\end{array}\right]^{T}$ and $\mathbf{A}$ is as in (14). A, $\mathbf{g}_{0}$ and $\mathbf{d}$ have dimensions $\left(\ell_{0}+1\right) \times\left(\ell_{0}+1\right)$, $\left(\ell_{0}+1\right) \times 1$ and $\left(\ell_{0}+1\right) \times 1$ respectively. By noting that all elements in $\mathbf{d}$ are zero except the last one, $g_{0}$ can be obtained by

$$
g_{0}(n)=\left(\frac{(-1)^{\ell_{0}+n+1}}{m}\right)\left(\frac{\triangle_{\left(\ell_{0}+1\right), n}}{\triangle}\right)
$$

where $\Delta_{i, j}$ and $\triangle$ are the cofactor of the $(i, j)$ element and the determinant of $\mathbf{A}$ respectively.

In summary, given $\ell_{0}, \omega_{p}, \omega_{S}$ and $m$, we pick $\ell_{1}$ using (13). Having fixed the orders of $G_{0}(z)$ and $G_{1}(z)$, we design the $m$-th band filter by iterative procedure described above. The spectral factor $\hat{G}(z)$ of $G(z)$ is thus $\hat{G}_{0}(z) G_{1}(z)$ where $\hat{G}_{0}(z)$ is the spectral factor of $G_{0}(z)$.

Example 9.1: We design a $3^{\text {rd }}$ band linear phase FIR filter of length 62 , with cutoff frequencies at $.2 \overline{3} \pi$ and $.4 \overline{3} \pi$. Here, $\ell_{0}=10$ and $\ell_{1}=21$. Fig. 1 shows the magnitude responses of $G(z)$ and $\hat{G}(z)$. $\hat{G}(z)$ has order 31 and has the largest possible number of zeros on the unit circle (under the constraint that $\hat{G}(z) \hat{G}\left(z^{-1}\right)$ is an $m$-th band filter.)

\section{Design of multistage decimation fil- ter}

We will present here an iterative procedure to design the multistage decimation filter [3] in fig. 2(a). Redrawing the structure as in fig. 2(b) for analysis purposes, we see that the effective transfer function to be designed has the form $H(z)=H_{0}(z) H_{1}\left(z^{2}\right) H_{2}\left(z^{4}\right)$. The filters $H_{0}(z)$ and $H_{1}(z)$ are required to be lowpass with minimum stopband energies so that aliasing is minimized. The passband requirements of $H_{0}(z)$ and $H_{1}(z)$ are not severe; however, the final stage $H_{2}(z)$ is required to be such that the overall system has a good passband (maximally flat or equiripple for example.) Suppose that the cutoff frequencies of $H(z)$ are $\omega_{p}=\frac{\pi}{4}-\epsilon$ and $\omega_{S}=\frac{\pi}{4}+\epsilon$, then the appropriate stopband edges of $H_{0}(z)$ and $H_{1}(z)$ are $\frac{\pi}{4}+\epsilon$ and $\frac{\pi}{2}+2 \epsilon$. Similarly, the passband edge of $H_{2}(z)$ is $\pi-4 \epsilon$. We will design the overall filter to have equiripple passband. We use an iterative method, where at each iteration we design one of the three filters $H_{0}(z), H_{1}(z), H_{2}(z)$ while the other two are held fixed at the 'current solution'. The procedure works as follows:

1. Initialize $H_{1}\left(e^{j \omega}\right)$ and $H_{2}\left(e^{j \omega}\right)$ to be unity. Design $H_{0}\left(e^{j \omega}\right)$ such that $\int_{\frac{\pi}{4}+c}^{\pi}\left|H_{0}\left(e^{j \omega}\right)\right|^{2} d \omega$ is minimized using the eigenfilter approach.

2. Fix $H_{0}\left(e^{j \omega}\right)$ (obtained from step 1) and $H_{2}\left(e^{j \omega}\right)$, and using the WE approach, design $H_{1}\left(e^{j \omega}\right)$ such that $\int_{\frac{\pi}{2}+2 \epsilon}^{\pi}\left|H_{0}\left(e^{j \frac{\omega}{2}}\right)\right|^{2}\left|H_{1}\left(e^{j \omega}\right)\right|^{2} d \omega$ is minimized.

3. Fix $H_{0}\left(e^{j \omega}\right)$ and $H_{1}\left(e^{j \omega}\right)$, design $H_{2}\left(e^{j \omega}\right)$ such that its passband $0 \leq \omega \leq \pi-4 \epsilon$ is equiripple using Remez exchange algorithm [10]. The weighting and desired functions for the Remez exchange algorithm are:

$$
\begin{gathered}
W\left(e^{j \omega}\right)=\left|H_{0}\left(e^{j \frac{\omega}{4}}\right)\right|\left|H_{1}\left(e^{j \frac{\omega}{2}}\right)\right| \\
D\left(e^{j \omega}\right)=\frac{1}{W\left(e^{j \omega}\right)} .
\end{gathered}
$$

4. Use the current $H_{1}\left(e^{j \omega}\right)$ and $H_{2}\left(e^{j \omega}\right)$, and design $H_{0}\left(e^{j \omega}\right)$ using the WE appoach such that

$$
\int_{\frac{\pi}{4}+\epsilon}^{\pi}\left|H_{1}\left(e^{j 2 \omega}\right)\right|^{2}\left|H_{2}\left(e^{j \epsilon \omega}\right)\right|^{2}\left|H_{0}\left(e^{j \omega}\right)\right|^{2} d \omega
$$

is minimized.

5. Fix $H_{0}\left(e^{j \omega}\right)$ and $H_{2}\left(e^{j \omega}\right)$, and design $H_{1}\left(e^{j \omega}\right)$ such that $\int_{\frac{\pi}{2}+2 \epsilon}^{\pi}\left|H_{0}\left(e^{j \frac{\omega}{2}}\right)\right|^{2}\left|H_{2}\left(e^{j 2 \omega}\right)\right|^{2}\left|H_{1}\left(e^{j \omega}\right)\right|^{2} d \omega$ is minimized using the WE approach.

6. Go to step 3 and repeat until the resulting $H(z)$ is satisfied.

The design procedure usually converges to a good solution after 5 iterations. By constraining the total energy of either $H_{0}(z)$ or $H_{1}(z)$ at the corresponding minimization step, the trivial solution is avoided.

Example 4.1 We design a multistage decimation filter with the orders of $H_{0}(z), H_{1}(z)$ and $H_{2}(z)$ to be 10,5 and 10 respectively. Thus, the overall filter has length 61 . Here, $\epsilon=0.05 \pi$. Fig. 3 shows the frequency responses of $H_{0}(z), H_{1}\left(z^{2}\right)$ and $H_{2}\left(z^{4}\right)$ respectively while fig. 4 shows the frequency responses of the overall filter $H(z)$.

\section{References:}

[1] P.P. Vaidyanathan and T.Q. Nguyen, "Eigenfilters: A new approach to least-squares FIR filter design and applications including Nyquist filters," IEEE Trans. on CAS, pp. 11-23, Jan. 1987. 
[2] F. Mintzer, ${ }^{\alpha}$ On half-band, third-band and $N$-th band FIR filters and their design," IEEE Trans. on ASSP, pp. 734-738, Oct. 1982.

[3] R. E. Crochiere and L. R. Rabiner, Multirate Digital Signal Processing, Englewood Cliffs, NJ, PrenticeHall, 1983.

[4] M. Renfors and T. Saramaki, "Recursive $N$-th band digital filters," (parts I and II), IEEE Trans. on CAS, pp. 24-51, Jan. 1987.

[5] P. P. Vaidyanathan, "Theory and design of $M$ channel maximally-decimated quadrature mirror filters with arbitrary $M$, having perfect reconstruction property”, IEEE Trans. on ASSP, pp. 476-492, April 1987.

[6] M. J. T. Smith and T. P. Barnwell III, “Exact reconstruction techniques for tree-structured subband coders", IEEE Trans. on ASSP, pp. 434-441, June 1986.

[7] F. Mintzer, "Filters for distortion-free two-band multirate filter banks", IEEE Trans. on ASSP, pp. 626630, June 1985.

[8] G. A. Mian and A. P. Nainer, "A fast procedure to design equiripple minimum-phase FIR filters”, IEEE Trans. on CAS, pp. 327-331, May 1982.

[9] B. Noble and J.W. Daniel, Applied Linear Algebra, Englewoods Cliffs, NJ., Prentice Hall, 1977.

[10] J.H. McClellan and T. Parks, “A unified approach to the design of optimum FIR linear phase digital filters, IEEE Trans. on Circuit Theory, pp. 697701, Nov. 1973.

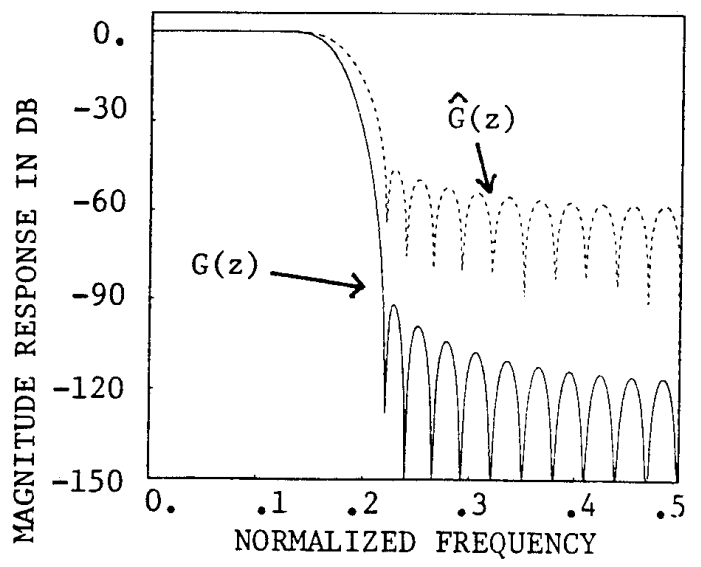

Fig. 1 Ex. 3.1 Magnitude responses of $G(z)$ and its spectral factor $\widehat{G}(z)$.

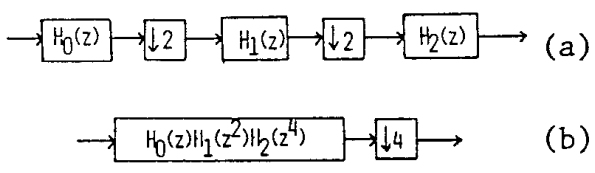

Fig. 2 The multistage decimation structure (a) The actual circuit (b) The redrawn version.

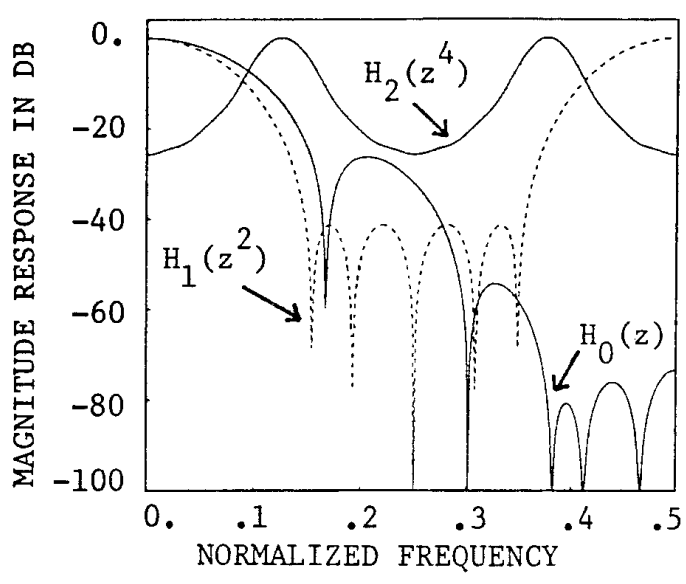

Fig. 3 Ex. 3.1 Magnitude responses of $\mathrm{H}_{0}(z), \mathrm{H}_{1}\left(\mathrm{z}^{2}\right)$ and $\mathrm{H}_{2}\left(z^{4}\right)$.

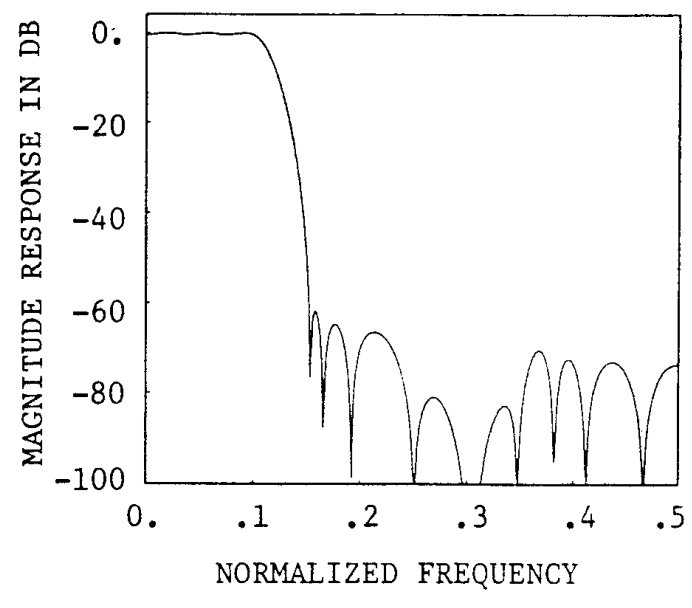

Fig. 4 Ex. 3.1 Magnitude response of $\mathrm{H}(z)$. 